\title{
Free Scattering Theory in Circularly Polarized Laser Field
}

\author{
Kishori Yadav, Jeevan Jyoti Nakarmi and Sanam Maharjan
}

Journal of Nepal Physical Society

Volume 4, Issue 1, February 2017

ISSN : 2392-473X

\section{Editors:}

Dr. Gopi Chandra Kaphle

Dr. Devendra Adhikari

Mr. Deependra Parajuli

JNPS, 4 (1), 78-87 (2017)

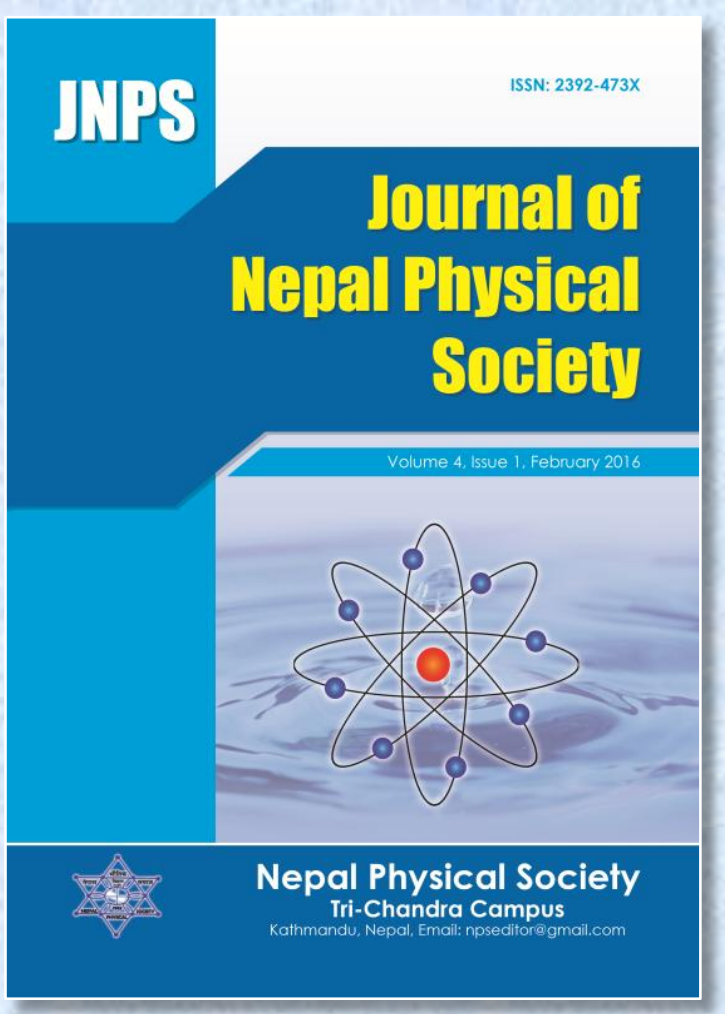

Published by:

Nepal Physical Society

P.O. Box : 2934

Tri-Chandra Campus

Kathmandu, Nepal

Email: npseditor@gmail.com 


\title{
Free Scattering Theory in Circularly Polarized Laser Field
}

\author{
Kishori Yadav ${ }^{1,2,}$, Jeevan Jyoti Nakarmi ${ }^{1}$ and Sanam Maharjan ${ }^{2}$ \\ ${ }^{1}$ Central Department of Physics, Tribhuvan University, Kirtipur, Nepal \\ ${ }^{2}$ Department of Physics, Patan Multiple Campus, Patan Dhoka \\ ${ }^{*}$ Corresponding Email: yadavkishori70@gmail.com
}

\begin{abstract}
In the present study, we have investigated scattering of an electron by hydrogen atoms in the presence of the Circularly Polarized (CP) laser field. We have discussed the polarization effect of laser field on hydrogen atom and effect of the resulted polarized potential on differential scattering cross section is studied. We assumed the scattered electrons having kinetic energy $100 \mathrm{eV}$ because it permitted to treat the scattering process in first order Born Approximation. The scattering electron was described by Volkov wave function. We found the differential scattering cross section decreases with the increase in scattering angle, for a fixed value of a laser parameters and kinetic energy of an incident electron. From this study we found that, the differential scattering cross section for the electric field perpendicular to the direction of momentum transfer depends on the elastic scattering amplitude. Finally, we concluded that the differential scattering cross section greatly depends upon the polarization of the laser field.
\end{abstract}

Keywords: Volkov Wave Function, Differential Scattering Creoss section, Polatization.

\section{INTRODUCTION}

Electron atom interaction in the presence of a laser field attracted considerable theoretical attention in the recent years not only because of the importance in applied areas (such as plasma heating or laser driven fusion), but also in view of their interest in fundamental atomic theory. The problem of this process, is in general, very complex, since in addition to the difficulties associated with the treatment of electron atom collision, the presence of the laser introduces new parameters (for example, the laser photon energy $\hbar \omega$ and intensity I) which may influence the collision. Moreover, the laser photon can play the role of a "third body" during the collision, and "dressed" the atomic states (Byron et al., 1987). It is therefore of interest to begin the theoretical analysis by considering the simpler problem of the scattering of an electron by a potential in the presence of a laser field. A fully realistic description of the target atom is quite difficult. We shall represent it here by a potential model. In the Pioneering work potential had been taken to be of the central self consistent type; a coulomb like at the origin $\left(V(r) \cong \frac{z}{r}\right)$. The laser field of a monochromatic infinite plane wave, linearly polarized, in the dipole approximation, the plane wave assumption is not critical, as the extension to the single mode laser pulse of adiabatically varying intensity can subsequently be made. At high intensities atomic transition abundantly involves multiphoton absorption and emission. The description by perturbation theory is no longer valid, and new method of solution of the Schrödinger equation are needed. A non perturbative theory was developed earlier by Kroll and Waston for low frequency regime (Kroll and Waston, 1973) is well suited for the range of the intense IR laser. In the following we shall present our theory for the low frequency regime. We shall mainly deal with the case of electron atom collision in the radiation field also termed free-free transitions. We shall first describe the formalism and then apply it to the case of a polarized potential.

The free -free process can theoretically be studied at various levels. As the target does not change states in this process, its own energy spectrum can be ignored and a simple potential can mimic the electron atom interaction. Furthermore, the collision can be treated as occurred at such intensities of electromagnetic field that the electron-field coupling is the dominant process and the target is transparent to the field such that photon-target coupling can be ignored. If, however, the frequency of the photon is such as to couple two stationary states of the target, then the target-field interaction becomes extremely important. Here we 
discussed such intensity of the electromagnetic field where the photon-field interaction can be neglected (Mason, 1993).

\section{THEORY}

We consider free-free transition for scattering of an electron by the potential;

$V(\mathbf{r}, t)=V(r)+\propto_{s} \frac{\vec{r} \cdot \vec{E}}{r^{3}}$

Which describe a hydrogen atom in a laser field . $\mathrm{V}(\mathrm{r})$ denotes the potential;

$V(r)=-e^{-2 r}\left(1+\frac{1}{r}\right)$

And $\propto_{s}$ is the static polarizability $\left(\propto_{s}=4.5\right.$ a.u. for hydrogen in its ground state). The second term in the equation (1) describes approximately the interaction between the electron and atomic dipole moment induced by the field.

For circularly polarized electric field in the dipole approximation

$\vec{E}(t)=i \frac{E_{o}}{2}\left[\exp (-i \omega t) \vec{\epsilon}-\exp (i \omega t) \overrightarrow{\epsilon^{*}}\right]$

Where $\vec{\epsilon}$ and $\vec{\epsilon}^{*}$ are polarization vectors. On expanding the exponential functions

we get

$\vec{E}(t)=\frac{E_{o}}{\sqrt{2}}\left\{\left[\cos (\omega t) \frac{i\left[\vec{\epsilon}-\vec{\epsilon}^{*}\right]}{\sqrt{2}}+\sin (\omega t) \frac{\left[\vec{\epsilon}+\vec{\epsilon}^{*}\right]}{\sqrt{2}}\right\}\right.$

Let us define two unit vectors along two orthogonal directions in the polarization plane:

$\widehat{e_{J}}=-\frac{i\left[\vec{\epsilon}-\vec{\epsilon}^{*}\right]}{\sqrt{2}}$

and

$\widehat{e_{l}}=\frac{\left[\vec{\epsilon}+\vec{\epsilon}^{*}\right]}{\sqrt{2}}$

$i \widehat{e_{j}}=\frac{\left[\vec{\epsilon}-\vec{\epsilon}^{*}\right]}{\sqrt{2}}$

Adding eqn. (5) \& (6),

$\widehat{e_{l}}+i \widehat{e_{J}}=\frac{\left[\vec{E}+\vec{\epsilon}^{*}\right]}{\sqrt{2}}+\frac{\left[\vec{E}-\vec{\epsilon}^{*}\right]}{\sqrt{2}}=\frac{2 \vec{\epsilon}}{\sqrt{2}}$

$\vec{\epsilon}=\frac{\widehat{e}_{\imath}+\widehat{l}_{J}}{\sqrt{2}}$

Subtracting eqn (5) \& (6),

$\widehat{e_{l}}-i \widehat{e}_{J}=\frac{\left[\vec{\epsilon}+\vec{\epsilon}^{*}\right]}{\sqrt{2}}-\frac{\left[\vec{\epsilon}-\vec{\epsilon}^{*}\right]}{\sqrt{2}}=\frac{2 \vec{\epsilon}^{*}}{\sqrt{2}}$

$\vec{\epsilon}^{*}=\frac{\widehat{e_{l}}-i \widehat{e_{J}}}{\sqrt{2}}$

So, the laser field given by the eqn (3), can be written as:

$\vec{E}(t)=\frac{E_{o}}{\sqrt{2}}\left[\widehat{e}_{\imath}(\sin \omega t)-\widehat{e}_{J}(\cos \omega t)\right]$

$E_{o}$ is amplitude and $\omega$ the frequency of the electric field; $\widehat{e_{l}}$ and $\widehat{e_{J}}$ are unit vectors along two orthogonal directions in the polarization plane.

$\vec{A}$ is the vector potential representing the laser field for an $\mathrm{CP}$ wave. Here we take $\vec{A}$ in spatially independent form because we have taken coulomb gauge.

We have from eqn (9),

$\vec{E}(t)=\frac{E_{o}}{\sqrt{2}}\left[\widehat{e}_{l}(\sin \omega t)-\widehat{e}_{J}(\cos \omega t)\right]$

On integrating over time, and substituting for $\vec{E}(t)$ from the eqn (9), we have

$\vec{A}=-c \int \frac{E_{o}}{\sqrt{2}}\left(\widehat{e}_{l} \sin \omega t-\widehat{e}_{J} \cos \omega t\right) d t$

$=c \frac{E_{o}}{\omega \sqrt{2}}\left[\widehat{e}_{l}(\cos \omega t)+\widehat{e}_{J}(\sin \omega t)\right]$

$\vec{A}=A_{o}\left[\widehat{e}_{l}(\cos \omega t)+\widehat{e}_{J}(\sin \omega t)\right.$

Where,

$$
A_{o}=\frac{c E_{o}}{\omega \sqrt{2}}
$$

Here we consider that the laser field as circularly polarized. Then we can change the Volkov wave function (Volkov, 1935) in a following simple form by substituting the value of the vector potential, we get,

$\chi(\vec{r}, t)=(2 \pi)^{\frac{-3}{2}} \exp \left\{i \vec{k} \cdot \vec{r}-\frac{i E_{k} t}{\hbar}\right\}$

$\exp \left\{-i \alpha_{o}\left[\widehat{e}_{l} \sin \omega t-\widehat{e}_{J} \cos \omega t\right] \cdot \vec{k}-\right.$

$\left.\frac{i}{\hbar} \int V(\vec{r}, t) d t\right\}$

Where,

$\alpha_{o}=\frac{e A_{o}}{m c \omega}$

as

$A_{o}=\frac{c E_{o}}{\omega \sqrt{2}}$

So, we have

$\alpha_{o}=\frac{e}{m c \omega} \frac{c E_{o}}{\omega \sqrt{2}}$

Hence,

$\frac{\sqrt{2}}{e} \alpha_{o}=\frac{E_{o}}{m \omega^{2}}$

Now, defining

$\vec{\alpha}(t)=\alpha_{o}\left[\widehat{e}_{l} \sin \omega t-\widehat{e}_{J} \cos \omega t\right]$

So eqn (10) becomes;

$\chi(\vec{r}, t)=(2 \pi)^{\frac{-3}{2}} \exp \left[(i \vec{k} \cdot \vec{r}-i \vec{k} \cdot \vec{\alpha}(t))-\frac{i E_{k} t}{\hbar}-\right.$

$\left.\frac{i}{\hbar} \int V(\vec{r}, t) d t\right]$ 
This is the required form of Volkov wave function.

Thus the first- order Born approximation, the Smatrix element corresponding to the scattering of

$$
S=\frac{-i}{\hbar} \int_{-\infty}^{\infty} d t<\chi_{\overrightarrow{k f}}|V(\vec{r}, t)| \chi_{\overrightarrow{k i}}>
$$

the electron on the potential given by,

Substituting for $\chi_{\overrightarrow{k f}}$ and $\chi_{\overrightarrow{k i}}$,

$$
\begin{aligned}
& S=\frac{-i}{\hbar(2 \pi)^{3}} \int_{-\infty}^{\infty} \int \exp \left(-i \vec{k} \cdot \vec{r}+i \overrightarrow{k_{f}} \cdot \vec{\alpha}(t)+i E_{k f} \frac{t}{\hbar}\right)\left\{\mathrm{V}(\mathrm{r})+\alpha_{\mathrm{s}} \frac{\overrightarrow{\mathrm{r}} \cdot \overrightarrow{\mathrm{E}}}{\mathrm{r}^{3}}\right\} \\
& \exp \left(i \vec{k}_{i} \cdot \vec{r}-i \vec{k}_{i} \cdot \vec{\alpha}(t)-i E_{k i} \frac{t}{\hbar}\right) d^{3} r d t
\end{aligned}
$$

Let the momentum transfer of the scattered electron be $\vec{q}=\overrightarrow{k_{l}}-\overrightarrow{k_{f}}$

$S=\frac{-i}{\hbar(2 \pi)^{3}} \int_{-\infty}^{\infty} \int \exp \{i \vec{q} \cdot \vec{r}\} \exp \{-i \vec{q} \cdot \vec{\alpha}(t)\} \exp \left\{i\left(E_{k f}-E_{k i}\right) \frac{t}{\hbar}\right\} \mathrm{V}(\mathrm{r}) d^{3} r d t$

$+\frac{-i}{\hbar(2 \pi)^{3}} \int_{-\infty}^{\infty} \int \exp \{i \vec{q} \cdot \vec{r}\} \exp \{-i \vec{q} \cdot \vec{\alpha}(t)\} \exp \left\{i\left(E_{k f}-E_{k i}\right) \frac{t}{\hbar}\right\}\left\{\alpha_{\mathrm{s}} \frac{\overrightarrow{\mathrm{r}} \cdot \overrightarrow{\mathrm{E}}}{\mathrm{r}^{3}}\right\} d^{3} r d t$

Considering the first part of the eqn. (14),

$S_{i f}^{B 1}=\frac{-i}{\hbar(2 \pi)^{3}} \int_{-\infty}^{\infty} \int \exp \{i \vec{q} \cdot \vec{r}\} \exp \{-i \vec{q} \cdot \vec{\alpha}(t)\} \exp \left\{i\left(E_{k f}-E_{k i}\right) \frac{t}{\hbar}\right\} \mathrm{V}(\mathrm{r}) d^{3} r d t$

$=f_{e l}^{B 1}(q) \frac{i}{\hbar(2 \pi)^{2}} \int_{-\infty}^{\infty} \exp \{-i \vec{q} \cdot \vec{\alpha}(t)\} \exp \left\{i\left(E_{k f}-E_{k i}\right) \frac{t}{\hbar}\right\} d t$

Where $\left.f_{e l}^{B 1}(q)=-(2 \pi)^{-1} \int \exp (i \vec{q} \cdot(\vec{r})) V(\vec{r})\right] d^{3} r$ is elastic scattering amplitude.

Taking,

$i \vec{q} \cdot \frac{e}{\sqrt{2}} \alpha_{o}\left[\widehat{e}_{l}(\sin \omega t)-\widehat{e_{J}}(\cos \omega t)\right]=i \frac{e}{\sqrt{2}} \alpha_{o}\left[\vec{q} \cdot \widehat{e_{l}}(\sin \omega t)-\vec{q} \cdot \widehat{e_{J}}(\cos \omega t)\right]$

$=i \frac{e}{\sqrt{2}} \alpha_{o} \sqrt{\left(\vec{q} \cdot \widehat{e_{l}}\right)^{2}+\left(\vec{q} \cdot \widehat{e_{J}}\right)^{2}}\left[\frac{\vec{q} \cdot \widehat{e_{l}}}{\sqrt{\left(\vec{q} \cdot \hat{e}_{l}\right)^{2}+\left(\vec{q} \cdot \widehat{e_{J}}\right)^{2}}}(\sin \omega t)-\frac{\vec{q} \cdot \widehat{e}_{J}}{\sqrt{\left(\vec{q} \cdot \widehat{e_{l}}\right)^{2}+\left(\vec{q} \cdot \widehat{e_{j}}\right)^{2}}}(\cos \omega t)\right]$

Let,

$\cos \phi_{q}=\frac{\vec{q} \cdot \widehat{e_{l}}}{\sqrt{\left(\vec{q} \cdot \widehat{e_{l}}\right)^{2}+\left(\vec{q} \cdot \widehat{e_{J}}\right)^{2}}}$ and $\sin \phi_{q}=\frac{\vec{q} \cdot \widehat{e_{J}}}{\sqrt{\left(\vec{q} \cdot \widehat{e_{l}}\right)^{2}+\left(\vec{q} \cdot \widehat{e_{J}}\right)^{2}}}$

and

$R_{q}=\frac{e}{\sqrt{2}} \alpha_{o} \sqrt{\left(\vec{q} \cdot \widehat{e_{l}}\right)^{2}+\left(\vec{q} \cdot \widehat{e_{J}}\right)^{2}}$

$\phi_{q}=\operatorname{arctg}\left(\frac{\left(\vec{q} \cdot \widehat{e_{J}}\right)}{\left(\vec{q} \cdot \widehat{e_{l}}\right)}\right)+l \pi$

Then,

$i \vec{q} \cdot \frac{e}{\sqrt{2}} \alpha_{o}\left[\widehat{e}_{l}(\sin \omega t)-\widehat{e}_{J}(\cos \omega t)\right]=i R_{q}\left[\cos \phi_{q}(\sin \omega t)-\sin \phi_{q}(\cos \omega t)\right]$

$=i R_{q} \sin \left(\omega t-\phi_{q}\right)$

So,

$S_{i f}^{B 1}=f_{e l}^{B 1}(q) \frac{i}{\hbar(2 \pi)^{2}} \int_{-\infty}^{t} d t \exp \left\{-i R_{q} \sin \left(\omega t-\phi_{q}\right)\right\} \exp \left\{i\left(E_{k f}-E_{k i}\right) \frac{t}{\hbar}\right\}$ 
We know the generating function of the Bessel Polynomial is (Harper, 2006)

$e^{i x \sin \phi}=\sum_{-\infty}^{\infty} J_{n}(x) e^{n i \phi}$

$\exp \left\{-i R_{q} \sin \left(\omega t-\phi_{q}\right)\right\}=\sum_{N=-\infty}^{\infty} J_{N}\left(\mathcal{R}_{q}\right) e^{(-i N \omega t)} e^{\left(i N \phi_{q}\right)}$

So

$S_{i f}^{B 1}=f_{e l}^{B 1}(q) \frac{i}{\hbar(2 \pi)^{2}} \int_{-\infty}^{t} d t \sum_{N=-\infty}^{\infty} J_{N}\left(\mathcal{R}_{q}\right) e^{(-i N \omega t)} e^{\left(i N \phi_{q}\right)} \exp \left\{i\left(E_{k f}-E_{k i}\right) \frac{t}{\hbar}\right\}$

Or,

$S_{i f}^{B 1}=f_{e l}^{B 1}(q) \frac{i}{\hbar(2 \pi)^{2}} \sum_{N=-\infty}^{\infty} J_{N}\left(\mathcal{R}_{q}\right) e^{\left(i N \phi_{q}\right)} \int_{-\infty}^{t} d t e^{(-i N \omega t)} \exp \left\{i\left(E_{k f}-E_{k i}-N \hbar \omega\right) \frac{t}{\hbar}\right\}$

In the atomic units $(\hbar=1)$

$S_{i f}^{B 1}=f_{e l}^{B 1}(q) \frac{i}{\hbar(2 \pi)^{2}} \sum_{N=-\infty}^{\infty} J_{N}\left(\mathcal{R}_{q}\right) e^{\left(i N \phi_{q}\right)} \hbar \int_{-\infty}^{t} d \frac{t}{\hbar} \exp \left\{i\left(E_{k f}-E_{k i}-N \hbar \omega\right) \frac{t}{\hbar}\right\}$

$=f_{e l}^{B 1}(q) \hbar \frac{i}{\hbar(2 \pi)^{2}} \sum_{N=-\infty}^{\infty} J_{N}\left(\mathcal{R}_{q}\right) e^{\left(i N \phi_{q}\right)} \delta\left(E_{k_{f}}-E_{k_{i}}-N \hbar \omega\right) 2 \pi$

$=f_{e l}^{B 1}(q) \frac{i}{2 \pi} \sum_{N=-\infty}^{\infty} J_{N}\left(\mathcal{R}_{q}\right) e^{\left(i N \phi_{q}\right)} \delta\left(E_{k_{f}}-E_{k_{i}}-N \hbar \omega\right)$

Here we have used properties of delta function[6];

$\delta\left(x-x^{\prime}\right)=\frac{1}{2 \pi} \int_{-\infty}^{\infty} e^{i k(x-x)} d k$

Or,

$\int_{-\infty}^{\infty} e^{i k(x-x)} d k=2 \pi \delta\left(x-x^{\prime}\right)$

Again the second part is;

$S_{i f}^{B 2}=\frac{-i}{\hbar(2 \pi)^{3}} \iint_{-\infty}^{t} \exp \{i \vec{q} \cdot(\vec{r})\} \exp \{-i \vec{q} \cdot \vec{\alpha}(t)\} \exp \left\{i\left(E_{k f}-E_{k i}\right) \frac{t}{\hbar}\right\}\left\{\alpha_{\mathrm{s}} \frac{\overrightarrow{\mathrm{r}} \cdot \overrightarrow{\mathrm{E}}}{\mathrm{r}^{3}}\right\} d^{3} r d t$

Since,

$\propto_{\mathrm{s}} \frac{\overrightarrow{\mathrm{r}} \cdot \overrightarrow{\mathrm{E}}}{\mathrm{r}^{3}}=\propto_{\mathrm{s}} \frac{E_{o}}{\mathrm{r}^{3}} \overrightarrow{\mathrm{r}} \cdot\left[\exp (-i \omega t) \vec{\epsilon}-\exp (i \omega t) \overrightarrow{\epsilon^{*}}\right]$

So,

$S_{i f}^{B 2}=\frac{-i}{\hbar(2 \pi)^{3}} \iint_{-\infty}^{t} \exp \{i \vec{q} \cdot(\vec{r})\} \exp \{-i \vec{q} \cdot \vec{\alpha}(t)\} \exp \left\{i\left(E_{k f}-E_{k i}\right) \frac{t}{\hbar}\right\}$

$\left\{\propto_{\mathrm{s}} \frac{E_{o}}{\mathrm{r}^{3}} \overrightarrow{\mathrm{r}} \cdot\left[\exp (-i \omega t) \vec{\epsilon}-\exp (i \omega t) \overrightarrow{\epsilon^{*}}\right]\right\} d^{3} r d t$

$-\left(\propto_{\mathrm{s}} E_{o}\right) \iint_{-\infty}^{t} \exp \{i \vec{q} \cdot(\vec{r})\} \exp \{-i \vec{q} \cdot \vec{\alpha}(t)\} \exp \left\{i\left(E_{k f}-E_{k i}\right) \frac{t}{\hbar}\right\}$

$\left.\left\{\exp (i \omega t) \frac{\overrightarrow{\mathrm{r}}}{\mathrm{r}^{3}} \cdot \overrightarrow{\in^{*}}\right\} d^{3} r d t\right]$

Considering first part of equation 19 
$\propto_{\mathrm{s}} E_{o} \iint_{-\infty}^{t} \exp \{i \vec{q} \cdot(\vec{r})\} \exp \{-i \vec{q} \cdot \vec{\alpha}(t)\} \exp \left\{i\left(E_{k f}-E_{k i}\right) \frac{t}{\hbar}\right\}$

$\left\{\exp (-i \omega t) \frac{\overrightarrow{\mathrm{r}}}{\mathrm{r}^{3}} \cdot \vec{\epsilon}\right\} d^{3} r d t$

$\left.=\propto_{\mathrm{s}} E_{o} \int \exp i \vec{q} \cdot(\vec{r})\right\} \frac{\overrightarrow{\mathrm{r}}}{\mathrm{r}^{3}} \cdot \vec{\epsilon} d^{3} r$

$\int_{-\infty}^{t} d t \exp \{-i \vec{q} \cdot \vec{\alpha}(t)\} \exp \left\{i\left(E_{k f}-E_{k i}\right) \frac{t}{\hbar}\right\} \exp (-i \omega t)$

$\left.=\propto_{\mathrm{s}} E_{o} \int \exp i \vec{q} \cdot(\vec{r})\right\} \frac{\overrightarrow{\mathrm{r}}}{\mathrm{r}^{3}} \cdot \vec{\epsilon} d^{3} r$

$\int_{-\infty}^{t} d t \sum_{N=-\infty}^{\infty} J_{N}\left(\mathcal{R}_{q}\right) e^{(-i N \omega t)} e^{\left(i N \phi_{q}\right)} \exp \left\{i\left(E_{k f}-E_{k i}\right) \frac{t}{\hbar}\right\} \exp (-i \omega t)$

Because,

$\exp \{-i \vec{q} \cdot \vec{\alpha}(t)\}=\sum_{N=-\infty}^{\infty} J_{N}\left(\mathcal{R}_{q}\right) e^{(-i N \omega t)} e^{\left(i N \phi_{q}\right)}$

Now,

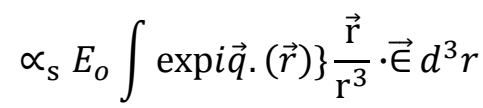

$\int_{-\infty}^{t} d t \sum_{N=-\infty}^{\infty} J_{N}\left(\mathcal{R}_{q}\right) e^{(-i N \omega t)} e^{\left(i N \phi_{q}\right)} \exp \left\{i\left(E_{k f}-E_{k i}\right) \frac{t}{\hbar}\right\} \exp (-i \omega t)$

$\left.=\propto_{\mathrm{s}} E_{o} \int \exp i \vec{q} \cdot(\vec{r})\right\} \frac{\overrightarrow{\mathrm{r}}}{\mathrm{r}^{3}} \cdot \vec{\epsilon} d^{3} r \sum_{N=-\infty}^{\infty} J_{N}\left(\mathcal{R}_{q}\right) e^{\left(i N \phi_{q}\right)}$

$\hbar 2 \pi \delta\left(E_{k_{f}}-E_{k_{i}}-(N+1) \hbar \omega\right)$

Replacing $\mathrm{N}$ by $\mathrm{N}-1$,

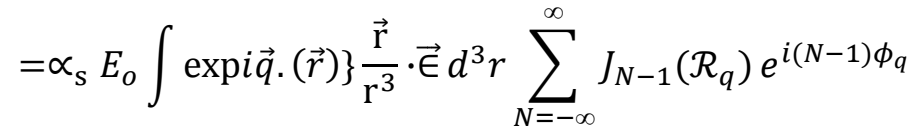

$\hbar 2 \pi \delta\left(E_{k_{f}}-E_{k_{i}}-N \hbar \omega\right)$

Here the integral;

$\int \frac{\vec{r} \cdot \vec{E}}{\mathrm{r}^{3}} \exp \{i \vec{q} \cdot(\vec{r})\} d^{3} r$

$=\iiint \frac{r \in \cos \theta}{\mathrm{r}^{3}} \exp \{i q r \cos \theta\} r^{2} d r \sin \theta d \theta d \varnothing$

$=-\frac{2 \pi \in \cos \theta}{i q}=-2 \pi \frac{\vec{q} \cdot \widehat{\epsilon}}{i q^{2}}$

Hence first part of eqn (19) is,

$=-(2 \pi)^{2} \hbar \propto_{\mathrm{s}} E_{o} \frac{\vec{q} \cdot \hat{\epsilon}}{q^{2}} \sum_{N=-\infty}^{\infty} J_{N-1}\left(\mathcal{R}_{q}\right) e^{i(N-1) \phi_{q}} \delta\left(E_{k_{f}}-E_{k_{i}}-N \hbar \omega\right)$ 
The second part of the eqn (19) is

$-\propto_{\mathrm{s}} E_{o} \iint_{-\infty}^{t} \exp \{i \vec{q} \cdot(\vec{r})\} \exp \{-i \vec{q} \cdot \vec{\alpha}(t)\} \exp \left\{i\left(E_{k f}-E_{k i}\right) \frac{t}{\hbar}\right\}$

$\left\{\exp (i \omega t) \frac{\overrightarrow{\mathrm{r}}}{\mathrm{r}^{3}} \cdot \overrightarrow{\epsilon^{*}}\right\} d^{3} r d t$

$=\iiint \frac{r \vec{\epsilon}^{*} \cos \theta}{r^{3}} \exp \{i q r \cos \theta\} r^{2} d r \sin \theta d \theta d \emptyset$

$=(2 \pi)^{2} \propto_{\mathrm{s}} E_{o} \frac{\vec{q} \cdot \vec{\epsilon}^{*}}{i q^{2}} \sum_{N=-\infty}^{\infty} J_{N}\left(\mathcal{R}_{q}\right) e^{\left(i N \phi_{q}\right)} \delta\left(E_{k_{f}}-E_{k_{i}}-(N-1) \omega\right)$

Replacing $\mathrm{N}$ by $\mathrm{N}+1$,

$=(2 \pi)^{2} \propto_{\mathrm{s}} E_{o} \frac{\vec{q} \cdot \vec{\epsilon}^{*}}{i q^{2}} \sum_{N=-\infty}^{\infty} J_{N+1}\left(\mathcal{R}_{q}\right) e^{\left(i N \phi_{q}\right)} \delta\left(E_{k_{f}}-E_{k_{i}}-N \hbar \omega\right)$

Thus with the help of the eqns.(20) \& (21), eqn.(19) becomes;

$S_{i f}^{B 2}=\frac{-i}{2 \pi} \delta\left(E_{k_{f}}-E_{k_{i}}-N \omega\right) e^{\left(i N \phi_{q}\right)}\left\{\propto_{\mathrm{s}} \frac{E_{o}}{\mathrm{q}}\left[e^{\left(-i \phi_{q}\right)} \frac{\vec{q} \cdot \vec{\epsilon}}{\mathrm{q}^{2}} J_{N-1}\left(\mathcal{R}_{q}\right)-e^{\left(i \phi_{q}\right)} \frac{\vec{q} \cdot \overrightarrow{\epsilon^{*}}}{\mathrm{q}^{2}} J_{N+1}\left(\mathcal{R}_{q}\right)\right]\right\}$

Thus we get;

$S=\frac{i}{2 \pi} \delta\left(E_{k_{f}}-E_{k_{i}}-N \omega\right) e^{\left(i N \phi_{q}\right)}\left\{f_{e l}^{B 1}(q) J_{N}\left(\mathcal{R}_{q}\right)\right.$

$\left.-\propto_{\mathrm{s}} \frac{\epsilon_{o}}{\mathrm{q}}\left[e^{\left(-i \phi_{q}\right)} \frac{\vec{q} \cdot \vec{\epsilon}}{\mathrm{q}^{2}} J_{N-1}\left(\mathcal{R}_{q}\right)-e^{\left(i \phi_{q}\right)} \frac{\vec{q} \cdot \overrightarrow{\epsilon^{*}}}{\mathrm{q}^{2}} J_{N+1}\left(\mathcal{R}_{q}\right)\right]\right\}$

$S_{i f}^{B 1}=\frac{i}{2 \pi} \delta\left(E_{k_{f}}-E_{k_{i}}-N \omega\right) f_{N}^{B 1}$

Where,

$f_{N}^{B 1}=e^{\left(i N \phi_{q}\right)}\left\{f_{e l}^{B 1}(q) J_{N}\left(\mathcal{R}_{q}\right)\right.$

$\left.-\propto_{\mathrm{s}} \frac{\epsilon_{o}}{\mathrm{q}}\left[e^{\left(-i \phi_{q}\right)} \frac{\vec{q} \cdot \vec{\epsilon}}{\mathrm{q}^{2}} J_{N-1}\left(\mathcal{R}_{q}\right)-e^{\left(i \phi_{q}\right)} \frac{\vec{q} \cdot \overrightarrow{\epsilon^{*}}}{\mathrm{q}^{2}} J_{N+1}\left(\mathcal{R}_{q}\right)\right]\right\}$

In the presence of radiation field the scattersd electron gain or loose energy equal to $N \omega$,such that $E_{f}=E_{i}+N \omega$ where $E_{i f}$ is the initial (final)energy of the projectile and $\mathrm{N}$ is the net number of photons exchanged (absorbed or emitted) by the colliding system and the CP field.The energy spectrum of the scattered electron therefore consists of the elastic term ,corresponding to $\mathrm{N}=0$ and a number of sidebands,each pair of sidebands corresponding to the same value of $|N|$.

We have differential scattering cross section is (Zettili, 2009)

$S_{i f}^{B 1}=\frac{i}{2 \pi} f_{N}^{B 1} \int_{-\infty}^{t} e^{i\left(E_{k_{f}}-E_{k_{i}}-N \omega\right) t} d t$

We know, $\lim _{\eta \rightarrow 0} e^{\eta t}=1$
We can insert this term in the above equation. So the equation becomes,

$S_{i f}^{B 1}=\frac{i}{2 \pi} f_{N}^{B 1} \lim _{\eta \rightarrow 0} \int_{-\infty}^{t} e^{\eta t} e^{i\left(E_{k_{f}}-E_{k_{i}}-N \omega\right) t} d t$

Now the transition probability is given by,

Transition probability $P_{i f=\left|S_{i f}^{B 1}\right|^{2}}$

$=\frac{1}{4 \pi^{2}}\left|f_{N}^{B 1}\right|^{2} \lim _{\eta \rightarrow 0} e^{2 \eta t} \frac{1}{\left(E_{k_{f}}-E_{k_{i}}-N \omega\right)^{2}+\eta^{2}}$;

$\left[|a+i b|^{2}=a^{2}+b^{2}\right]$

Similarly the transition rate is,

$\frac{d P_{i f}}{d t}=\frac{1}{2 \pi^{2}}\left|f_{N}^{B 1}\right|^{2} \lim _{\eta \rightarrow 0} \frac{e^{2 \eta t} \eta}{\left(E_{k_{f}}-E_{k_{i}}-N \omega\right)^{2}+\eta^{2}}$ 
We have the relation,

$\lim _{\eta \rightarrow 0} \frac{e^{2 \eta t} \eta}{\omega_{n l}^{2}+\eta^{2}}=\pi \hbar \delta\left(E_{n}-E_{l}\right)$

$\delta\left(\hbar \omega_{n}-\hbar \omega_{l}\right)=\frac{1}{\hbar} \delta\left(\omega_{n}-\omega_{l}\right)$

Using this, $\frac{d P_{i f}}{d t}=\frac{1}{2 \pi^{2}}\left|f_{N}^{B 1}\right|^{2} \delta\left(\omega_{k_{f}}-\omega_{k_{i}}-N \omega\right)$

$=\frac{1}{2 \pi^{2}}\left|f_{N}^{B 1}\right|^{2} \delta\left(E_{k_{f}}-\gamma\right)$

where,

$\gamma=E_{k_{i}}+N \omega$

$=\frac{-1}{2 \pi^{2}}\left|f_{N}^{B 1}\right|^{2} \frac{m}{k} \delta\left(k_{f}-k\right)$

Now the total transition from an incident momentum state $k_{i}$ into a solid angle $d \Omega$ is,

$W=\frac{1}{2 \pi^{2}} \int\left|f_{N}^{B 1}\right|^{2} \frac{m}{k} \delta\left(k_{f}-k\right) k^{2} \frac{1}{(2 \pi)^{3}} d k d \Omega$

We have, $\sum_{k} \rightarrow \frac{1}{(2 \pi)^{3}} \int d^{3} k$

$W=\frac{m}{(2 \pi)^{4}}\left|f_{N}^{B 1}\right|^{2} d \Omega \int \delta\left(k_{f}-k\right) k d k$

$W=\frac{m}{(2 \pi)^{4}}\left|f_{N}^{B 1}\right|^{2} k_{f} d \Omega$

$\int \delta\left(k_{f}-k\right) d k=1$ for $k=k_{f}$

Where, $k_{f}$ is the momentum of the scattered particle. If the velocity of the incident particle is $v$,

Where, $v=\frac{\hbar k_{i}}{m} \quad$ then the differential cross section $d \sigma$ is given by ;

$d \sigma=\frac{\text { total transition rate }}{\text { flux of incident particles }}$

$d \sigma=\frac{W}{j_{\text {inc }}}$

Where,

$j_{\text {inc }}=\frac{\hbar}{2 m i}(\psi * \nabla \psi-\psi \nabla \psi *)$

Where $\psi$ is a plane wave function and $\psi *$ is a complex wave function.

Substituting the value of plane wave function in this equation we get,

$j_{\text {inc }}=\frac{\hbar k_{i}}{m}$
So,

$$
\begin{gathered}
d \sigma=\frac{m}{(2 \pi)^{4}}\left|f_{N}^{B 1}\right|^{2} k_{f} \frac{m}{\hbar k_{i}} d \Omega \\
\frac{d \sigma}{d \Omega}=\frac{m^{2}}{(2 \pi)^{4}} \frac{k_{f}}{k_{i}}\left|f_{N}^{B 1}\right|^{2} \\
f_{N}^{B 1}=e^{\left(i N \phi_{q}\right)}\left\{f_{e l}^{B 1}(q) J_{N}\left(\mathcal{R}_{q}\right)\right. \\
\quad \propto_{\mathrm{s}} \frac{E_{o}}{\mathrm{q}^{2}}|\vec{q} \cdot \vec{\epsilon}|\left[J_{N-1}\left(\mathcal{R}_{q}\right)\right. \\
\left.\left.\quad-J_{N+1}\left(\mathcal{R}_{q}\right)\right]\right\}
\end{gathered}
$$

Now,

$J_{N}^{\prime}\left(\mathcal{R}_{q}\right)=\frac{\left[J_{N-1}\left(\mathcal{R}_{q}\right)-J_{N+1}\left(\mathcal{R}_{q}\right)\right]}{2}$

$f_{N}^{B 1}=e^{\left(i N \phi_{q}\right)}\left\{f_{e l}^{B 1}(q) J_{N}\left(\mathcal{R}_{q}\right)-2 \propto_{\mathrm{s}} \frac{E_{o}}{\mathrm{q}^{2}} \mid \vec{q}\right.$.

$\left.\vec{E} \mid J^{\prime}{ }_{N}\left(\mathcal{R}_{q}\right)\right\}$

Thus equation (27) becomes

$\frac{d \sigma_{N}^{C P}}{d \Omega}=\frac{m^{2}}{(2 \pi)^{4}} \frac{k_{f}}{k_{i}} \mid\left\{f_{e l}^{B 1}(q) J_{N}\left(\mathcal{R}_{q}\right)-2 \propto_{\mathrm{s}} \frac{E_{o}}{\mathrm{q}^{2}} \mid \vec{q}\right.$.

$\left.\vec{\epsilon} \mid J^{\prime}{ }_{N}\left(\mathcal{R}_{q}\right)\right\}\left.\right|^{2}$

Equation (30) is the differential scattering cross section in terms of elastic transition amplitude.

Here, $f_{e l}^{B 1}(q)=\frac{2\left(q^{2}+8\right)}{\left(4+q^{2}\right)^{2}} \quad($ Yadav and Nakarmi, 2015)

$\vec{q}=\overrightarrow{k_{l}}-\overrightarrow{k_{f}}$

$\mathcal{R}_{q}=\alpha_{0}|\vec{\epsilon} \cdot \vec{q}|$

$J_{N}^{\prime}\left(\mathcal{R}_{q}\right)=\frac{\left[J_{N-1}\left(\mathcal{R}_{q}\right)-J_{N+1}\left(\mathcal{R}_{q}\right)\right]}{2}$

\section{RESULTS AND DISCUSSION}

In the present study, we have studied the elastic scattering of an electron-atom interaction by absorbing photons from the Circularly Polarized (CP) laser field. We have considered hydrogen atom and effect of polarized potential in scattering is studied by considering high electron energy of $100 \mathrm{eV}$ and laser field of moderate intensities i.e. $E_{0}=10^{8} \mathrm{~V} / \mathrm{cm}$ and $\omega=2 \mathrm{eV}$.

We have calculated the differential scattering cross section as;

$\frac{d \sigma_{N}^{C P}}{d \Omega}=\frac{m^{2}}{(2 \pi)^{4}} \frac{k_{f}}{k_{i}} \mid\left\{f_{e l}^{B 1}(q) J_{N}\left(\mathcal{R}_{q}\right)-2 \propto_{\mathrm{s}} \frac{E_{o}}{\mathrm{q}^{2}} \mid \vec{q}\right.$.

$\left.\vec{\in} \mid J^{\prime}{ }_{N}\left(\mathcal{R}_{q}\right)\right\}\left.\right|^{2}$

From the above relation it is clear that non linear differential scattering cross section depends on number of photons $(\mathrm{N})$ and the momentum transfer 
of incident electron (q). In atomic unit mass of electron is considered unity.

Here we have studied the nature of Bessel $\mathbf{J}$ with $\mathrm{q}$, variation of elastic scattering amplitude with $\mathrm{q}$ and above equation is plotted as a function of $\mathrm{q}$ and scattering angle and the results are shown below;

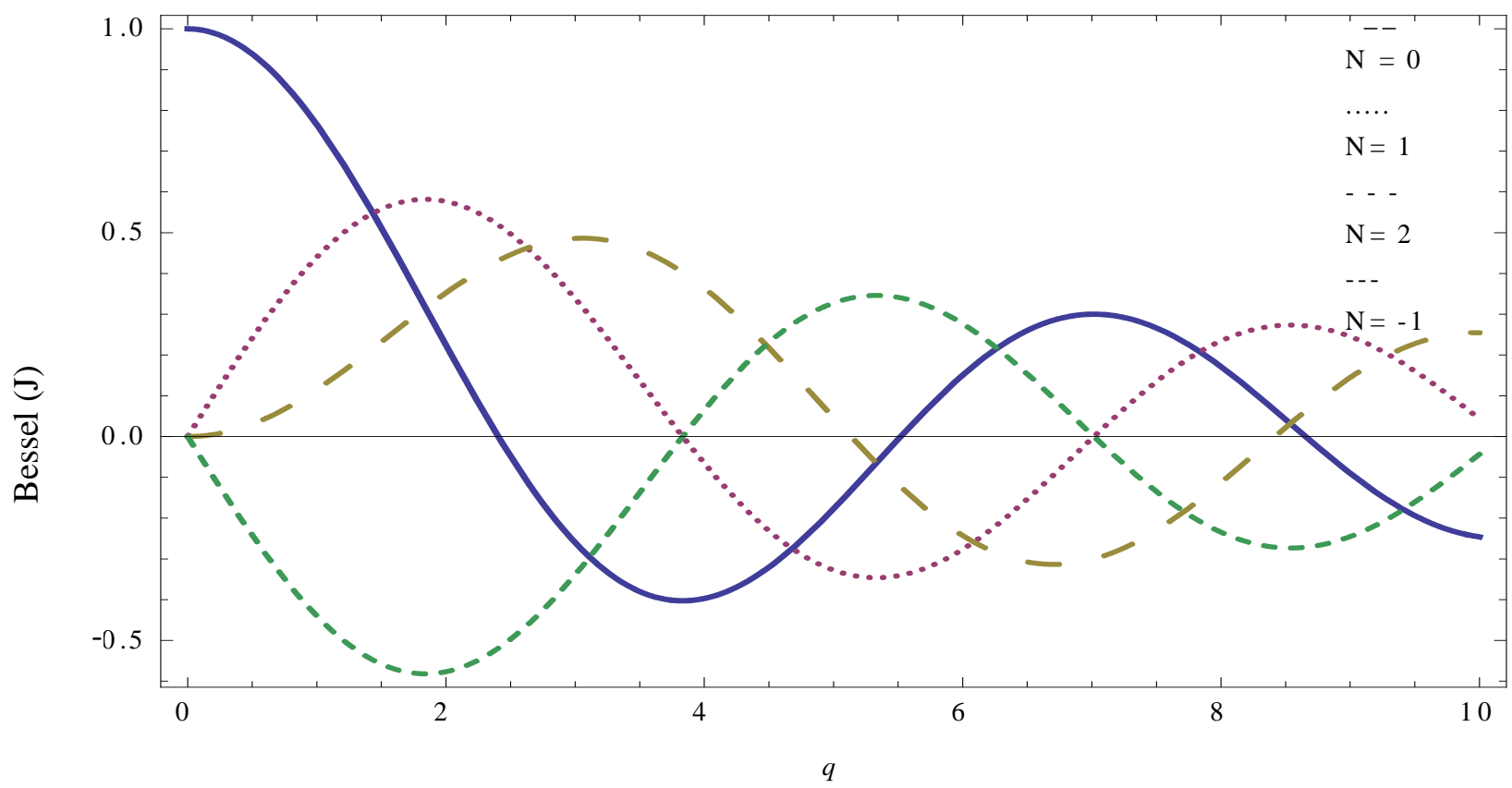

Fig.1. Variation of Bessel J with Momentum Transfer (q) for (a) $N=0,(b) N=1,(c) N=2$ and (d) $N=-1$.

Above figure 1 shows the variation of Bessel $\mathrm{J}$ with momentum transfer (q) for number of photons exchanged.

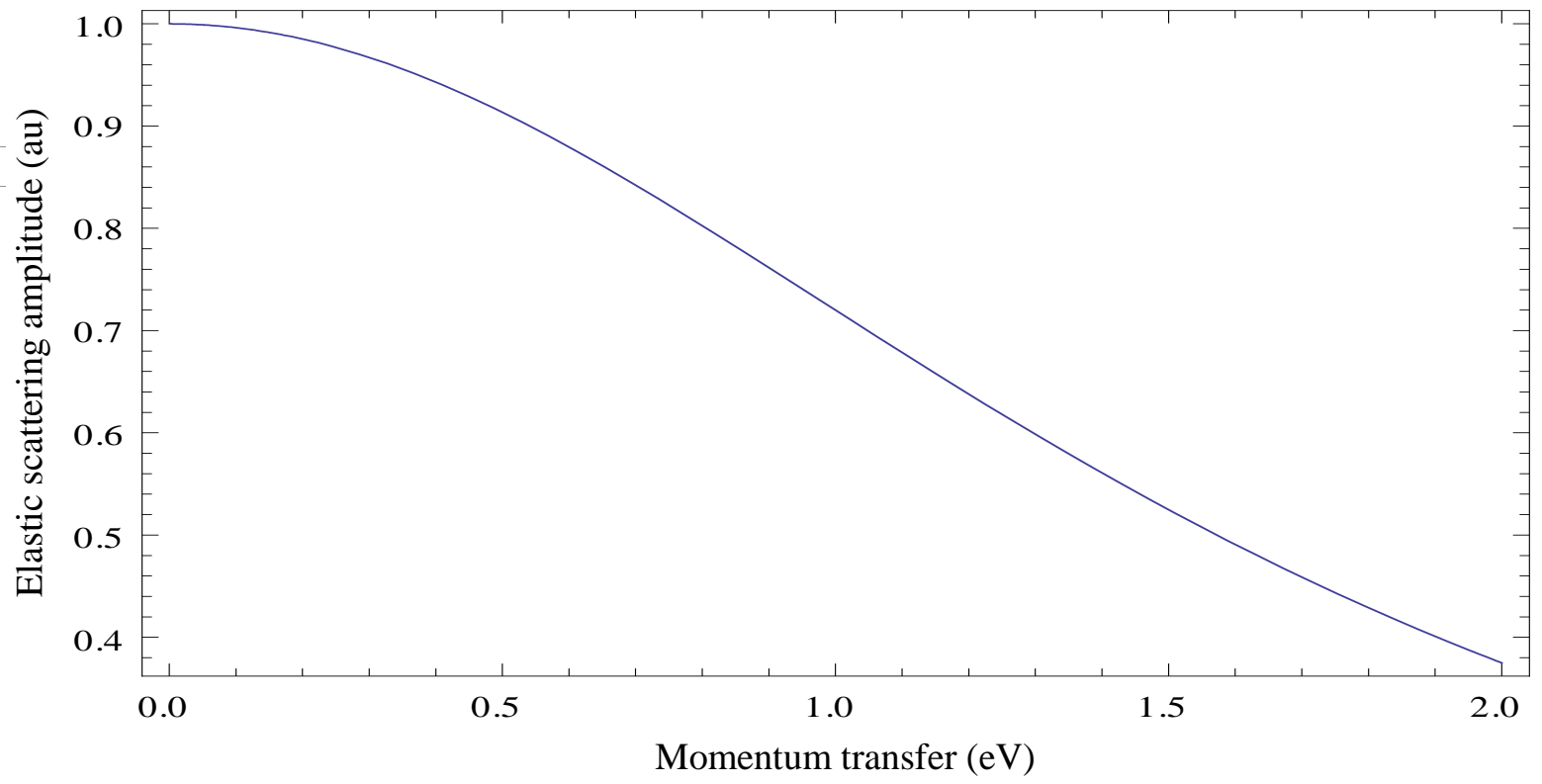

Fig.2. Variation of Elastic scattering amplitude with the momentum transfer (q).

From the plot, figure 2, we see that the elastic scattering amplitude decreases as the momentum transfer of the incident electron increases. Going on increasing the momentum transfer of an incident electron we will get zero value of elastic scattering amplitude. 


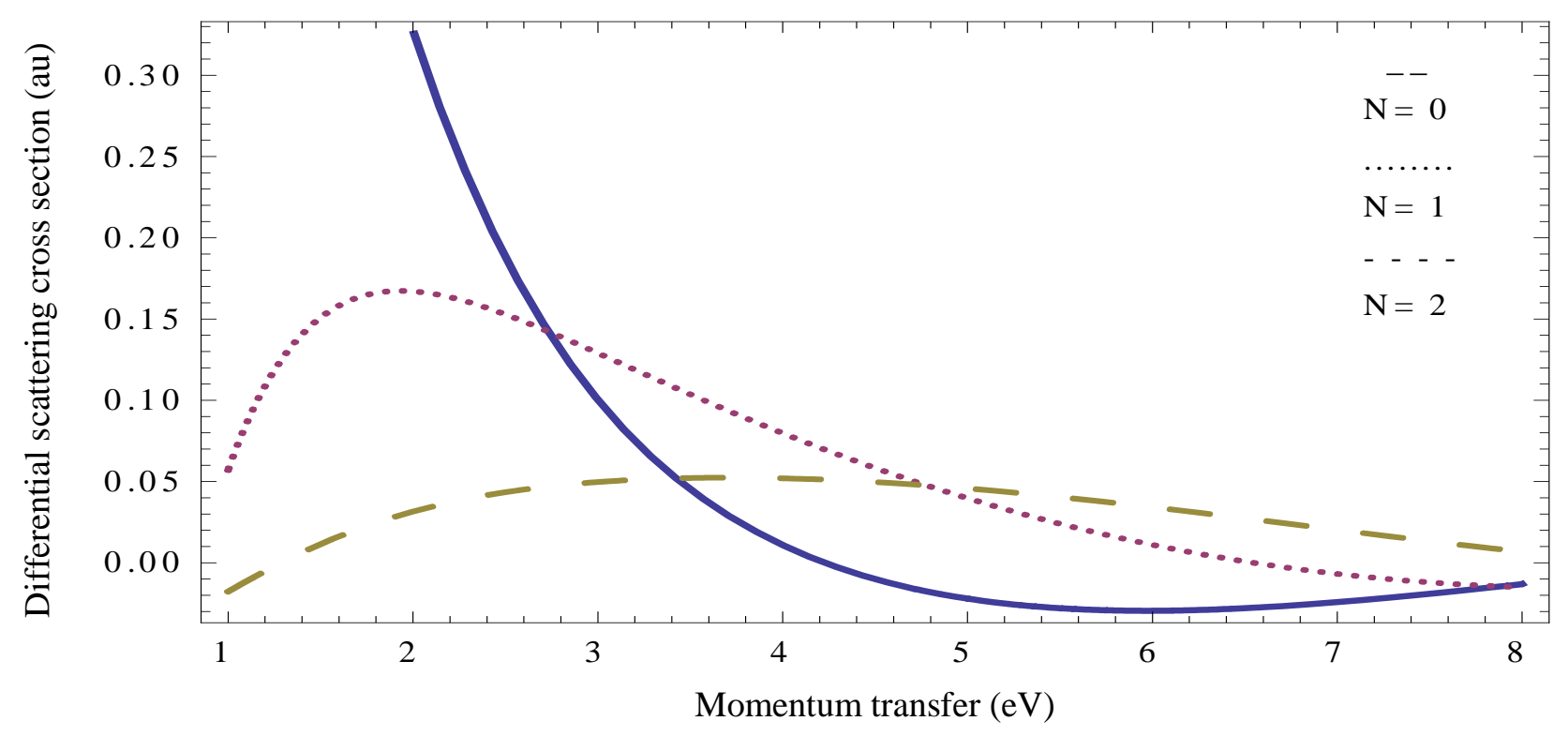

Fig.3. Variation of differential cross section with the momentum transfer for (a) $N=0,(b) N=1,(c) N=2$

From the plot, figure 3, we see that the differential scattering cross section decreases sharply as the momentum transfer of the incident electron increases then remains fairly constant for some value and then increases for $\mathrm{N}=0$. But for
$\mathrm{N}=1$ and $\mathrm{N}=2$ differential scattering cross section first increases with increase in $\mathrm{q}$ and then decreases as in plot shown. For $\mathrm{N}=1$ decrease is sharp than for $\mathrm{N}=2$.

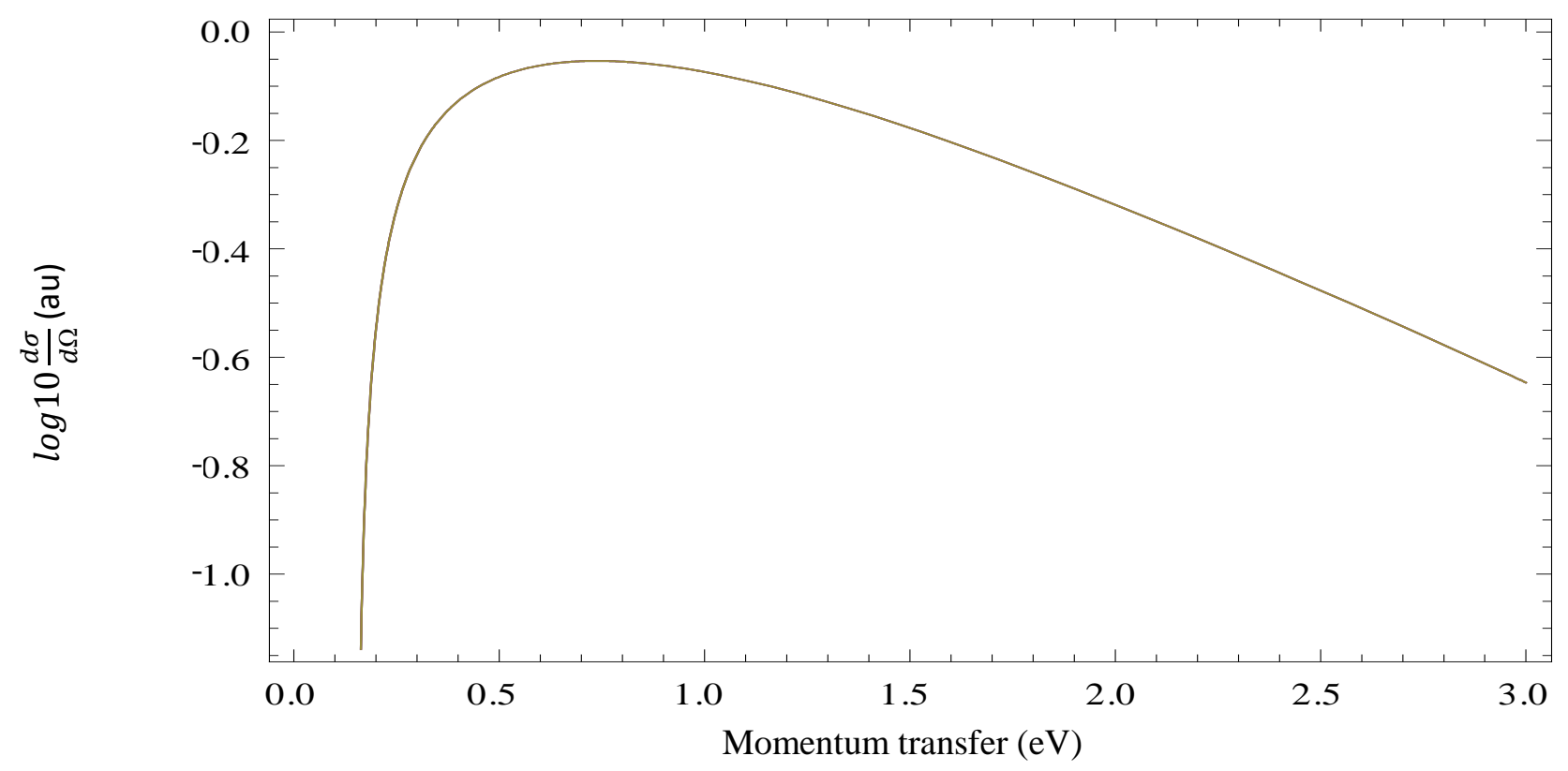

Fig.4. Variation of differential Scattering cross section (in log) with the momentum transfer (q).

From the plot, figure 4, we see that the differential scattering cross section increases for small range with increase in momentum transfer (q) and then decreases with further increase in $\mathrm{q}$.

\section{CONCLUSIONS}

It is generally observed that when electrons are scattered from the atom in the presence of a laser field, a new effect is observed which are not 
accessible in ordinary electron-atom scattering. This collision have the basic peculiarity of being processes in which three subsystem are present (i) the electron (ii) the target atom (iii) the radiation field. The last one provided energy and momentum and is characterized by the polarization of its electric field, which introduces in this collision process a new physical axis.

In this thesis work, we have investigated scattering of an electron by hydrogen atoms in the presence of the Circularly Polarized (CP) laser field. In our present work we have included the polarization effect of laser field on hydrogen atom and effect of the resulted polarized potential on differential scattering cross section is studied. Since we assumed the scattered electrons to have initially 100 $\mathrm{eV}$ kinetic energy, this permitted us to treat the scattering process in first order Born Approximation. The scattering electron was described by Volkov wave function. During the derivation of differential scattering cross section, we have used the proper definition of phases in Graf's addition theorem of Bessel functions (Watson, 1992). This work is outlined in this work. From this study we concluded that the differential scattering cross section of an electron depends upon the momentum transfer i.e. kinetic energy of incident electron and strength of the laser field where as in ordinary electron-atom scattering, cross section only depends upon the scattering angle .For fixed scattering angle, the differential scattering cross section increases with increase in momentum transfer (q) for short range but it decreases with further increase in momentum transfer. The decrease in differential scattering cross section with increase in momentum transfer (q) after certain range is more prominent in the order of no photon, single photon and two-photon. The differential scattering cross section decreases with the increase in scattering angle, for a fixed value of a laser parameters and kinetic energy of an incident electron. Also from this study we see that, the differential scattering cross section for the electric field perpendicular to the direction of momentum transfer depends on the elastic scattering amplitude. So from this study we concluded that the differential scattering cross section is also greatly depends upon the polarization of the laser field.

\section{ACKNOWLEDGEMENT}

NAST, Nepal and UGC, Nepal are acknowledged for partial financial support to the project.

\section{REFERENCE}

Byron, F. W.; Francken, P., and Joachain, C. J. (1987). Laser assisted elastic electron atom collision, J. Phys: B: At. Mol. Phys. 20:54875503.

Harper, C. (2006). Introduction to Mathematical physics, Department of Physics California State University, Hayward, Prentice Hall of India Pvt. Ltd., New Delhi.

Kroll N. M., and Waston, K. M. (1973). Phys. Rev. A, 8:804.

Mason, N. J. (1993). Laser assisted electron- atom collision, Rep. Prog. Phys., 56:1275-1346.

Volkov, D. M. (1935). J. Phys., 94:250.

Watson, G. N. (1922). Theory of Bessel Function, Cambridge at the University Press.

Yadav, K., and Nakarmi, J. J. (2015). Free Free scattering theory of the elastic scattering of an atom. International Journal of Physics, 3:32-39.

Zettili, N. (2009). Quantum Mechanics Concepts and Application, second edition, John Wiley and Sons, Ltd. 